Verification of the CNGS timing system using fast diamond detectors

This content has been downloaded from IOPscience. Please scroll down to see the full text. 2013 JINST 8 P01017

(http://iopscience.iop.org/1748-0221/8/01/P01017)

View the table of contents for this issue, or go to the journal homepage for more

Download details:

IP Address: 137.138.125.163

This content was downloaded on 08/07/2014 at 07:48

Please note that terms and conditions apply. 


\title{
Verification of the CNGS timing system using fast diamond detectors
}

\author{
H. Jansen, ${ }^{a, 1}$ P. Alvarez Sanchez, ${ }^{a}$ S. Bart Pedersen, ${ }^{a}$ B. Dehning, ${ }^{a}$ D. Dobos, ${ }^{a}$ \\ E. Effinger, ${ }^{a}$ A. Ferrari, ${ }^{a}$ E. Griesmayer, ${ }^{b}$ E. Gschwendtner, ${ }^{a}$ I. Kozsar, ${ }^{a}$ \\ D. Missiaen, ${ }^{a}$ H. Pernegger, ${ }^{a}$ P.R. Sala, ${ }^{c}$ J. Serrano ${ }^{a}$ and C. Ward ${ }^{d}$ \\ ${ }^{a} C E R N$, \\ Geneva, Switzerland \\ ${ }^{b}$ CIVIDEC Instrumentation, \\ Vienna, Austria \\ ${ }^{c}$ INFN - Sezione di Milano, \\ Milano, Italy \\ ${ }^{d}$ Imperial College London, \\ London, U.K. \\ E-mail: Hendrik.Jansen@cern.ch
}

ABSTRACT: A new fast diagnostic tool was installed in the CNGS facility in 2011 following the neutrino time-of-flight results published by OPERA in September 2011. Among others, four polycrystalline CVD (pCVD) diamond detectors were placed in the secondary beam line about $1200 \mathrm{~m}$ downstream of the CNGS target in order to measure the beam structure of the muons which are produced together with the muon neutrinos. Upstream of the CNGS target, a fast beam current transformer measures the proton beam structure. The sub-nanosecond single-pulse time resolution of pCVD diamond for a minimum ionising particle in combination with a GPS system allows the measurement of the GPS timing of individual secondary particle bunches crossing these detectors with a precision of $<1 \mathrm{~ns}$. The complicated structure of the CNGS muon beam in 2011 necessitates the combination of adjacent bunches in order to compare the proton beam structure with the muon beam structure. An analysis of the detector signals was carried out, which provides an independent timing measurement at CERN with a precision of $1.2 \mathrm{~ns}$. Uncertainties from other sources as cable lengths add up to $3.4 \mathrm{~ns}$, resulting in an overall precision of $3.6 \mathrm{~ns}$. The distance between the beam current transformer and the diamond detectors has been measured to $(1859.95 \pm 0.02) \mathrm{cm}$. The nominal time-of-flight of $(6205.3 \pm 1.7) \mathrm{ns}$ for a $17 \mathrm{GeV} / \mathrm{c}$ muon, as present in the CNGS muons beam, falls within the uncertainties of the measured time-of-flight of $(6205.2 \pm 3.6)$ ns. Hence, the GPS timing measurements performed at CERN are consistent.

KEYWORDS: Diamond detectors; Timing detectors; Materials for solid-state detectors

\footnotetext{
${ }^{1}$ Corresponding author.
} 


\section{Contents}

1 Introduction 1

2 The CNGS facility 2

3 Experimental set-up 3

3.1 Diamond detectors 3

3.2 Electronics, read-out and charge collection 5

4 Flux simulation 6

5 Trigger and timing $\quad 7$

$\begin{array}{lll}5.1 & \text { Survey } & 7\end{array}$

5.2 Set-up at CERN 8

5.2.1 Primary beam 8

$\begin{array}{llr}\text { 5.2.2 Secondary beam } & 9\end{array}$

$\begin{array}{ll}5.3 \text { Calculation of } \delta_{\text {delay }} & 10\end{array}$

6 Data analysis $\quad 10$

6.1 Detector response 11

$\begin{array}{ll}6.2 & \text { Signal to noise ratio } \\ 6.3 & 12\end{array}$

$\begin{array}{ll}\text { 6.3 Time resolution and phase shift } & 13\end{array}$

$\begin{array}{lll}6.4 & \text { Data treatment } & 14\end{array}$

$\begin{array}{lll}\text { 6.4.1 Averaging } & 14\end{array}$

$\begin{array}{lll}\text { 6.4.2 Filtering and normalisation } & 14\end{array}$

$\begin{array}{ll}\text { 6.5 Calculation of } \delta_{\text {offset }} & 15\end{array}$

6.5.1 The local method 16

$\begin{array}{ll}\text { 6.5.2 The global method } & 16\end{array}$

$\begin{array}{lll}7 & \text { Results } & 19\end{array}$

8 Conclusion $\quad 20$

\section{Introduction}

The CNGS facility (CERN Neutrinos to Gran Sasso) [1] aims at the direct detection of muonneutrino to tau-neutrino oscillation by measuring the appearance of the tau-neutrino. An intense muon-neutrino beam is generated at CERN and directed over $732 \mathrm{~km}$ towards the Gran Sasso National Laboratory (LNGS) in Italy, where four detectors (BOREXINO [2], ICARUS [3], LVD [4], OPERA [5]) are located. In addition to the main goal of tau-neutrino measurements, the facility 
profits from accurate time and distance measurements between the source of the CNGS neutrino beam and the Gran Sasso experiments, allowing the determination of the neutrino velocity with high accuracy. Until 2011 the start time of the neutrinos racing towards Gran Sasso has been solely based on measurements of the fast beam current transformer that is installed in the primary proton beam line upstream the CNGS target; the time structure of the protons passing through the beam current transformer gives the Global Positioning System (GPS) time tag. After the publication from the OPERA collaboration in September 2011 on the neutrino velocity measurements [6], several polycrystalline chemical vapour deposition (pCVD) diamond detectors (DD) were placed in the secondary beam line about $1200 \mathrm{~m}$ downstream of the CNGS target in order to measure the time structure of the muon spills independently. This allows an accurate measurement of the GPS timing of the muon bunches crossing these detectors, and provides an independent timing measurement at CERN.

\section{The CNGS facility}

The $400 \mathrm{GeV} / c$ CNGS proton beam is fast extracted from the CERN SPS accelerator; during a $6 \mathrm{~s}$ long cycle there are two extractions separated by $50 \mathrm{~ms}$, each lasting $10.5 \mu \mathrm{s}$ with up to $2.4 \cdot 10^{13}$ protons/extraction. With a $5 \mathrm{~ns}$ bunch spacing resulting from the $200 \mathrm{MHz}$ SPS RF system, the number of bunches per extraction is 2100 . The beam is sent down an $840 \mathrm{~m}$ long proton beam line onto a carbon target producing kaons and pions. The positively charged pions and kaons are energy-selected and guided with two focusing lenses, the so-called horn and reflector, in the direction of Gran Sasso. These particles decay in a $1000 \mathrm{~m}$ long, $2.5 \mathrm{~m}$ diameter vacuum tube into muon-neutrinos and muons. All the hadrons, i.e. protons that have not interacted in the target and pions/kaons that have not decayed in flight, are absorbed in a hadron stopper, see "hadron stop" in figure 1. Only neutrinos and muons can traverse this $18 \mathrm{~m}$ long block of graphite and iron. The muons, which are ultimately absorbed downstream in abound $500 \mathrm{~m}$ of rock, are measured in two muon detector stations, each consisting of 41 ionisation chambers. The stations reside in two caverns called Pit 1 and Pit 2 which are separated by $67 \mathrm{~m}$ of rock. The average energy of the muon-neutrinos which are sent to Gran Sasso, is $17 \mathrm{GeV} / c$. A schematic overview of the CNGS neutrino beam facility at CERN is shown in figure 1. The time information at CNGS for the OPERA neutrino time-of-flight (ToF) analysis was derived from a fast beam current transformer (BCTF40) [7] that is installed in the CNGS extraction line upstream of the CNGS target. The time structure of the extracted protons is measured with this BCTF40 detector and the time information is tagged with a GPS time.

Polycrystalline CVD (pCVD) diamond detectors were installed in the muon pits in November 2011. Both the measurement set-up for the BCTF40 and the one for the DDs are connected to a General Machine Timing (GMT) receiver, which communicates with the GPS system. The muon beam structure is measured via the DDs and is also GPS time tagged. Using both time tags and taking into account all additional delays, the timing measurement at CERN can be verified independently.

The next section describes the experimental set-up, followed by the description of the flux simulation. Details of the trigger and timing system are explained in section 5. The data analysis is described in section 6 . 


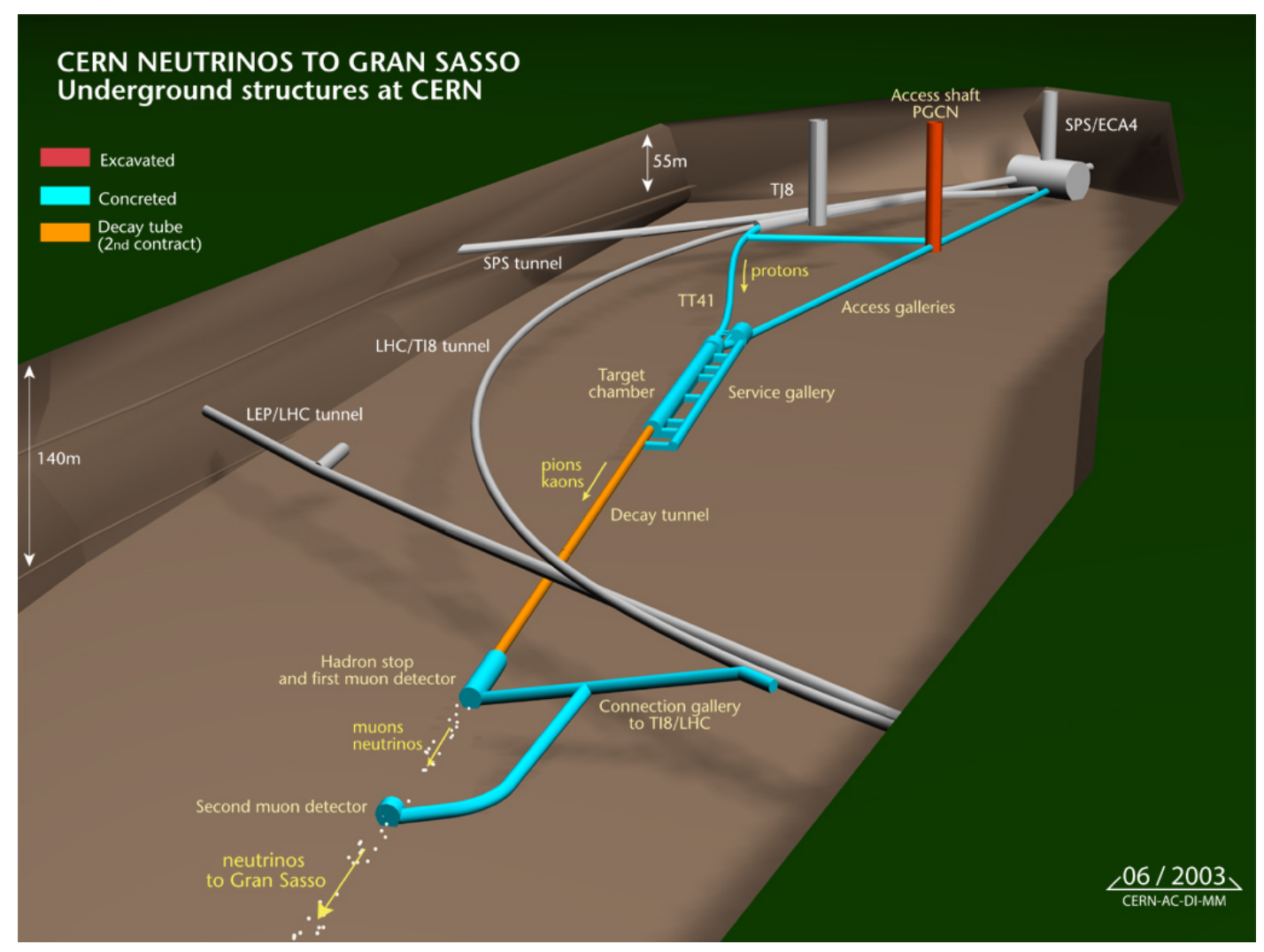

Figure 1. Schematic overview of the CNGS neutrino beam facility at CERN.

\section{Experimental set-up}

\subsection{Diamond detectors}

CVD diamond is a suitable detector material for the measurement of the time structure of the CNGS muon beam, as the detector response to a narrow muon bunch is a signal with full width half maximum (FWHM) of approximately $2 \mathrm{~ns}$. This is sufficiently smaller than the bunch spacing $(5 \mathrm{~ns})$ of the CNGS muon beam, and hence the signals stemming from adjacent bunches are sufficiently separated in order to identify a rising edge for each pulse. Note that the time resolution of diamond detectors is even better, i.e. it is in the sub-nanosecond regime, as will be shown in section 6 . Short signal time is realised by operating the detectors at $1 \mathrm{~V} / \mu \mathrm{m}$. The high free charge carrier mobility in diamond of $\sim 800 \mathrm{~cm}^{2} / \mathrm{Vs}$ at $1 \mathrm{~V} / \mu \mathrm{m}$ results in a first order estimation of the FWHM of

$$
t_{\mathrm{FWHM}}=\frac{\ln (2) * \mathrm{CCD}}{v_{\mathrm{drift}}}=\frac{\ln (2) * \mathrm{CCD}}{\mu E} \approx 1.7 \mathrm{~ns},
$$

where CCD is the charge collection distance, and a value of CCD $=200 \mu \mathrm{m}$ is used here. Hence, the FWHM is $\leq 2 \mathrm{~ns}$.

The measured signal is composed of a DC part of $10.5 \mu$ s length and is modulated by a $5 \mathrm{~ns}$ structure resulting from the SPS RF. The DC part is the result of the overlay of signal tails from adjacent bunches. 

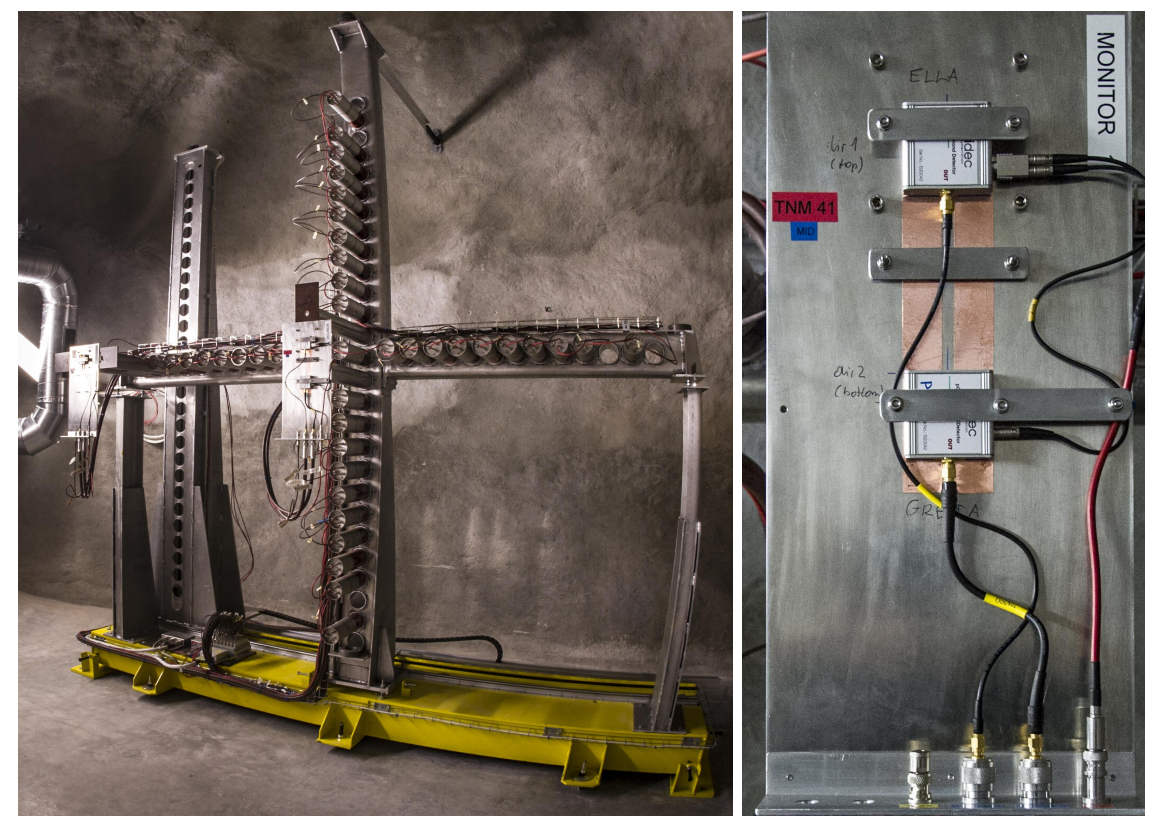

Figure 2. Photo of the muon detector station (left) in Pit 1 with two detectors in the middle of the cross and one on the left end. Additionally, a close-up of CT and CB is shown (right).

Table 1. Main parameters of the diamond detectors installed in muon Pit 1.

\begin{tabular}{|c|c|c|c|}
\hline Detector name & Centre Top (CT) & Centre Bottom (CB) & Left (L) \\
\hline Specified thickness $[\mu \mathrm{m}]^{1}$ & 500 & 500 & 500 \\
\hline Electrode size $^{1}$ & $\varnothing=3 \mathrm{~mm}$ & $8 \times 8 \mathrm{~mm}^{2}$ & $8 \times 8 \mathrm{~mm}^{2}$ \\
\hline Electrode area $\left[\mathrm{mm}^{2}\right]^{1}$ & 7.1 & 64 & 64 \\
\hline Ionisation volume $\left[\mathrm{mm}^{3}\right]^{2}$ & 3.53 & 32.0 & 32.0 \\
\hline Detector capacitance $[\mathrm{pF}]^{2}$ & 3 & 10 & 10 \\
\hline Time constant $[\mathrm{ps}]^{2}$ & 150 & 500 & 500 \\
\hline Charging capacitance $[\mathrm{nF}]^{1}$ & 11 & 2 & 11 \\
\hline
\end{tabular}

${ }^{1}$ Data specified by manufacturer. $\quad{ }^{2}$ Calculated from manufacturer data.

Three pCVD diamond detectors were installed in Pit 1, two at the centre, called Centre Top (CT) and Centre Bottom (CB) and one $135 \mathrm{~cm}$ left of the centre (L). A forth one is installed in Pit 2. In this work the signals from the DDs in Pit 1 are discussed. The set-up in Pit 1 is depicted in figure 2 (left), and details of the Centre DDs in figure 2 (right).

The sensor material was produced by Element Six [11] and metallised by Diamond Detectors Ltd [12]. DDL quotes a thickness of $500 \mu \mathrm{m}$ and a CCD of $200 \mu \mathrm{m}$. However, a smaller charge collection distance of about $180 \mu \mathrm{m}$ seems to be present in the sensor material. All main DD parameters are listed in table 1. The final detectors, including a sensor holder, an RF-tight housing, and a PCB with $50 \Omega$ read-out lines, were provided by Cividec Instrumentation [13]. 


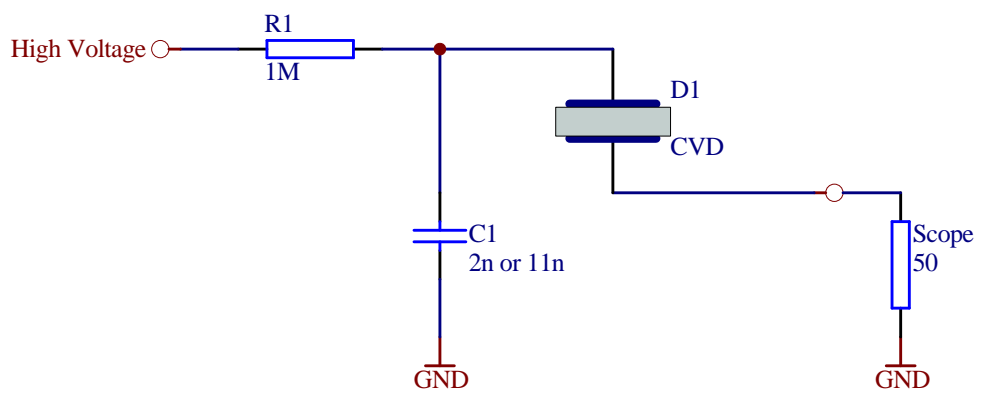

Figure 3. Connection scheme of the detectors. R1 is the loading resistor for the bias voltage, $\mathrm{C} 1$ the charging capacitor, and D1 the detector.

\subsection{Electronics, read-out and charge collection}

The detectors were connected to the bias voltage via a loading resistor (R1) of $1 \mathrm{M} \Omega$ resistance and a charging capacitor (C1), see figure 3 and table 1 . The signals were read out at the lowvoltage side of the detector, which was DC-terminated to ground by a $1 \mathrm{M} \Omega$ resistor. The charging capacitors were of $11 \mathrm{nF}$ capacitance for $\mathrm{CT}$ and $\mathrm{L}$, and of $2 \mathrm{nF}$ capacitance for $\mathrm{CB}$. All detectors were operated at $500 \mathrm{~V}$, i.e. $1 \mathrm{~V} / \mu \mathrm{m}$. It is important to mention, that the detectors were read out directly without electronic amplification. The low detector capacitances of $10 \mathrm{pF}$ and $3 \mathrm{pF}$ in combination with the $50 \Omega$ read-out impedance provide fast time constants of $500 \mathrm{ps}$ and $150 \mathrm{ps,}$ respectively. This allows for the resolution of the $5 \mathrm{~ns}$ RF structure from the extracted SPS beam, which has already been proven [14].

Two LeCroy WaveRunner 104MXi-A oscilloscopes with an analogue bandwidth of $1 \mathrm{GHz}$ and a sampling frequency of $5 \mathrm{GS} / \mathrm{s}$ were used for the data read-out. The oscilloscopes sample the incoming analogue signals with 8 bits, providing an analogue to digital converter (ADC) range of 256. All detectors were connected to the read-out oscilloscopes via CK50 cables [15]. The $50 \Omega$ cables have a specified attenuation per $100 \mathrm{~m}$ of $9.1 \mathrm{~dB}$ at $1 \mathrm{GHz}$ and $2.7 \mathrm{~dB}$ at $100 \mathrm{MHz}$ and a velocity factor (VF) of $78 \%$, VF being the speed of wave propagation in the cable relative to the speed of light in vacuum. The transmission time of the three CK50 cables was measured using an oscilloscope and a pulser set to output few nanosecond long pulses with a rise time of $500 \mathrm{ps}$ on $50 \Omega$ at a repetition rate of $1 \mathrm{kHz}$. The one-way transmission times are $328.1 \mathrm{~ns}, 328.3 \mathrm{~ns}$ and $328.1 \mathrm{~ns}$ for CT, CB, and L, respectively, corresponding to the actual cable length of about $77 \mathrm{~m}$. The trigger signals of the oscilloscopes were calibrated within a precision of $0.5 \mathrm{~ns}$.

The charge collection of all detectors were checked prior to installation in the pit at two different energies. One test was done using an ${ }^{241} \mathrm{Am} \alpha$-source, and the other with a ${ }^{90} \mathrm{Sr} \beta$-source. The detectors showed a charge collection of $(135,000 \pm 15 \%)$ electrons/alpha, which fits the expected charge resulting from a sealed ${ }^{241} \mathrm{Am} \alpha$-source $\left(E_{\alpha}=4.7 \mathrm{MeV}\right)$ for pCVD diamond with a CCD $=190 \mu \mathrm{m}$. The spread over the three detectors is due to differing CCDs. The charge tests based on the $\beta$-source showed a charge collection of $(6500 \pm 20 \%)$ electrons/MIP across the detectors, compatible with a CCD $=180 \mu \mathrm{m}$.

The expected collected charge per extraction from the high intensity muon beam is $Q=Q_{\mathrm{MIP}} *$ $I_{\mu}$, where $I_{\mu}$ is the muon flux integrated over the ionisation volume and over the whole extraction. 
The measured charge per extraction, averaged over the three detectors, agrees at a $15 \%$ level with simulations, indicating a linear response of the diamond even at this high particle fluence. The dark current prior to the extractions is of the order of few $\mathrm{nA}$, and hence negligible.

\section{Flux simulation}

Monte Carlo simulations were exploited to assess the feasibility of the measurement and the detector working environment. Calculations were performed with the FLUKA [8] code by slightly modifying the standard set-up already used for all CNGS related simulations [9]. The reliability of this simulation framework has been verified on many observables, in particular for what concerns the response of the ionisation chambers in the muon pits [10]. No effort to describe the exact geometry of detectors and supports was performed at this stage, since the scope of the calculation was essentially to determine the time distribution of all particles that generate a signal in the diamond detectors in order to assess that this time distribution does not affect the sensitivity of the measurement. Not only muons, but also secondary electrons and photons generated by muons in the pit walls and internal materials were accounted for. The diamond detectors were modelled as a uniform layer sandwiched between two $\mathrm{Al}$ supporting layers of $3 \mathrm{~mm}$ thickness. The detectors thickness has been indicatively set at $0.1 \mathrm{~mm}$ (simulations were performed before the actual choice of the detectors), with a density of $3.52 \mathrm{~g} / \mathrm{cm}^{3}$. In the simulation, two such sandwiches were inserted in each of the muon pits, one near to the entrance wall and one in front of the ionisation chambers, oriented perpendicularly to the beam and spanning the whole transverse dimension of the pit. The energy deposition in the detectors were simulated as a function of the arrival time of particles and as a function of radial distance with respect to the beam axis. The intrinsic resolution and timing response of the detectors have not been included in the simulations. For both positions in each pit, the distribution of deposited energy versus arrival time peaks at the arrival of the muon bunch. The falling edge of the simulated signal drops two orders of magnitudes in about $2 \mathrm{~ns}$. Very small secondary peaks are present, with amplitudes five orders of magnitude lower than the prompt peak, as shown in figure 4 for a single zero-width muon bunch. The time delay of these secondary peaks is consistent with the travel time of particles reaching the detectors after being reflected by the downstream pit walls. Convolution of the response to a single bunch with the time structure of a beam consisting of 2100 bunches shows that the original beam time structure is only minimally affected.

Particle fluences and deposited energy at the actual diamond detectors positions are reported in table 2 per $1 \cdot 10^{13}$ protons on target (pot). The contribution from charged hadrons and neutrons is negligible. Energy deposition values have been rescaled linearly to the finally used detector thickness in order to get a first order estimate of the needed dynamic range of the oscilloscope for data taking. For a single extraction with $2.4 \cdot 10^{13}$ protons, an energy of $1.2 \mathrm{TeV}$ are deposited in CB. With an average electron-hole-pair creation energy of $13.5 \mathrm{eV}$ and a CCD of $200 \mu \mathrm{m}$ the amount of the total measured charge per extraction is about $56 \mathrm{nC}$. An average bunch creates therefore $27 \mathrm{pC}$. If it is assumed, that these charges drift for $1 \mathrm{~ns}$, they induce a current of $27 \mathrm{~mA}$, and hence a voltage difference of $1.3 \mathrm{~V}$ appears at the input of the oscilloscope. With a set gain of $500 \mathrm{mV} /$ division, the oscilloscope translates the $1.3 \mathrm{~V}$ to about $83 \mathrm{ADCs}$, which can as well be seen in figure 10 , section 6 . 


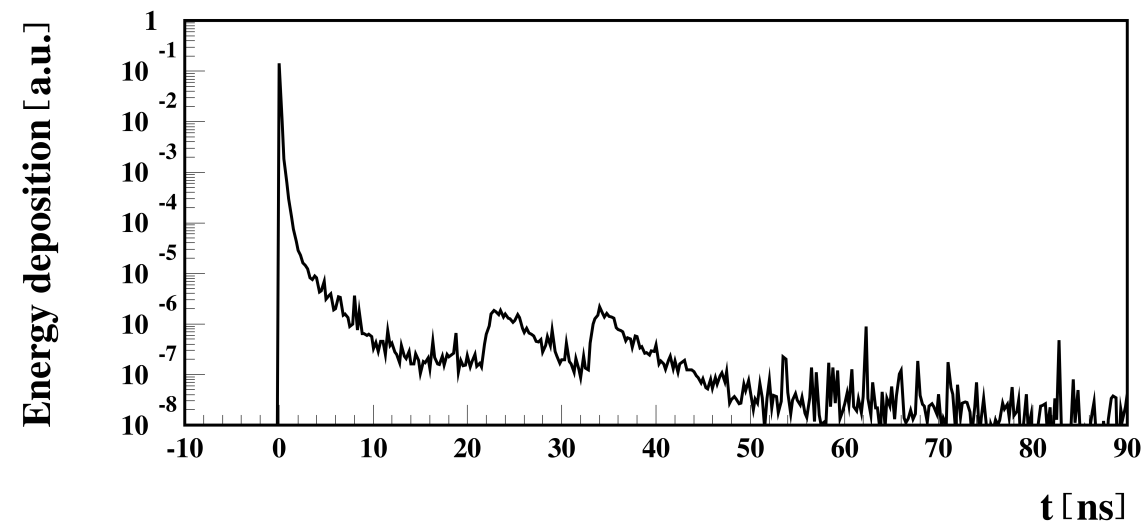

Figure 4. Time distribution of the energy deposition for a diamond detector in Pit 1.

Table 2. Particle fluencies and deposited energy at the diamond detectors positions for $1 \cdot 10^{13}$ pot.

\begin{tabular}{|l|c|c|c|c|}
\hline Position & $\begin{array}{r}\mu \text { flux } \\
\text { part/cm } / \mathrm{cm}^{2} / 10^{13}\end{array}$ pot & $\begin{array}{c}\text { Dep. energy } \\
\mathrm{GeV} / \mathrm{cm}^{2} / 10^{13} \text { pot }\end{array}$ \\
\hline Pit 1, centre & $2.4 \cdot 10^{7}$ & $2.7 \cdot 10^{6}$ & $1.5 \cdot 10^{7}$ & 7750 \\
Pit 1, left & $2.3 \cdot 10^{6}$ & $8.6 \cdot 10^{5}$ & $5.5 \cdot 10^{6}$ & 1250 \\
\hline
\end{tabular}

\section{Trigger and timing}

To confirm the accuracy of the CNGS timing system it is necessary to relate the timing signals from the DDs with those from the BCTF40 detector previously used. This is achieved by finding the ToF between the two detector points using the measured timestamps and relative offsets, and ensuring that they agree with the nominal ToF over the same distance. In order for the ToF to be measured on the nanosecond scale it is necessary to know precisely all the delays introduced between the detection of a particle in the detector and the signal being registered by the system in addition to the distance travelled by the particle. The transmission times of all data-carrying cables were measured using a variety of methods, including reflectometry and using a transportable CS4000 caesium clock [17]. The distance travelled was measured by an independent survey group.

\subsection{Survey}

Initially, the survey group has aligned all the components of the CNGS line, including the BCTF40, with respect to a network of geodetic points which was determined by topographical methods linked to the SPS geodetic network. Neither GPS measurements at the surface level nor link between surface to tunnel were realised. The relative accuracy of this underground network, measured with high precision instruments such as the Electronic Distance Measurer Mekometer ME5000, the total station TDA5005 and the Gyro-theodolite GYROMAT2000, could be estimated to be in the range of few millimetre. Then, additional measurements were carried out, with less accurate measuring techniques, to localise the DDs and the BCTF40 position with respect to the same network. 


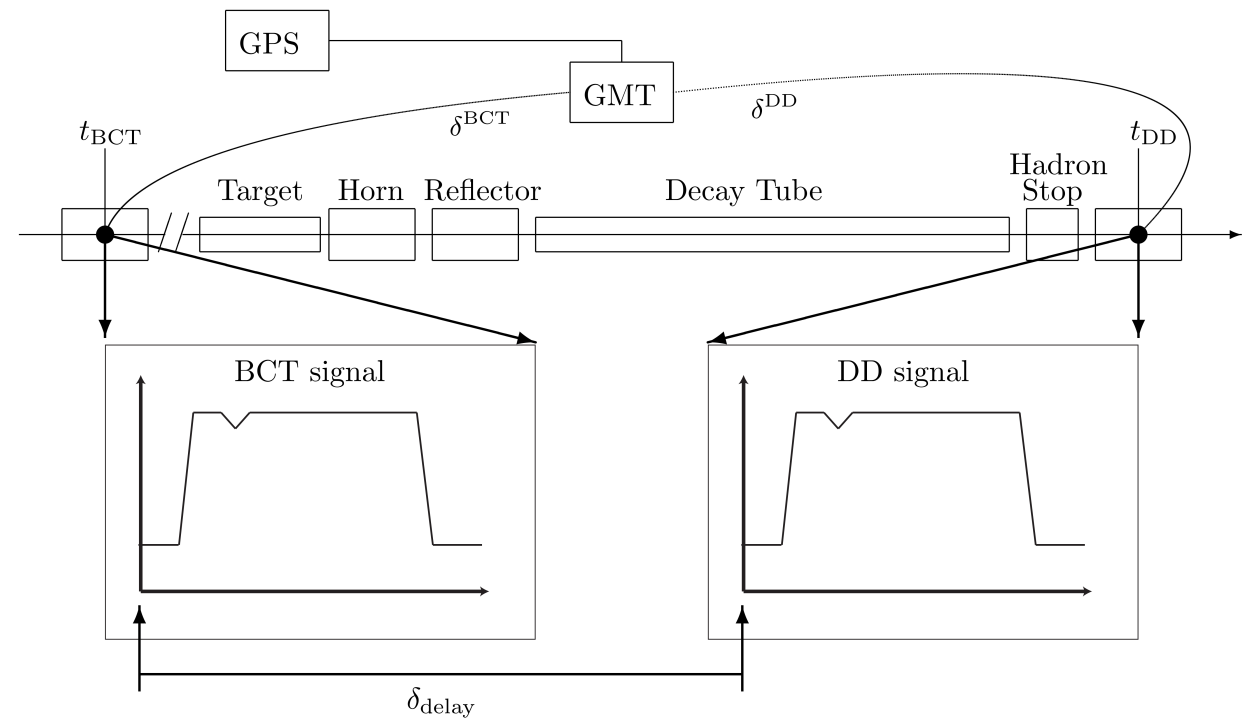

Figure 5. Overview of timing offsets at the CNGS facility. The distance between the BCTF40 and the DDs is about $1860 \mathrm{~m}$.

These measurements yield a distance $d_{\mathrm{BCT}-\mathrm{DD}}$ between the BCTF40 and the DDs in Pit 1 of:

$$
d_{\mathrm{BCT}-\mathrm{DD}}=(1859.95 \pm 0.02) \mathrm{m} \text {. }
$$

\subsection{Set-up at CERN}

There are two timing measurements made on the CNGS beam line; one for the primary (proton) beam as it leaves the SPS ring and passes BCTF40 and one for the secondary (muon and neutrino) beam from the DDs located in Pit 1, see figure 5. The delays examined here are those between the start time of an event window as measured by a reference GMT receiver located in the CERN control room (CCR) and the same time measured at the oscilloscopes reading the detector signals. Hence, this is done for the BCTF40 and for the DDs individually, with a common "clock" provided by the GMT. Additionally, the cable length for the signal transmission from the detectors to the oscilloscopes is taken into account. The different delays for the primary and the secondary beam are explained subsequently.

\subsubsection{Primary beam}

There are four individual values which sum to create the delay $\delta^{\mathrm{BCT}}$ for the primary beam time stamp: (1) the time taken to traverse the hardware between the BCTF40 and the oscilloscope in room HCA442, (2) between the oscilloscope and the a so-called Control Timing Receiver (CTR1) in HCA442, (3) between the CTR1 in HCA442 and the reference CTR in the CCR, and (4) a value of $99216.0 \mathrm{~ns}$ subtracted, via software, from the database time stamp as a correction between the CTR time and the GPS time. These values sum to

$$
\delta^{\mathrm{BCT}}=(-581.0 \pm 0.9+26.6 \pm 1.0+10077.8 \pm 2.0-99216.0) \mathrm{ns}=(-89692.6 \pm 2.4) \mathrm{ns}
$$




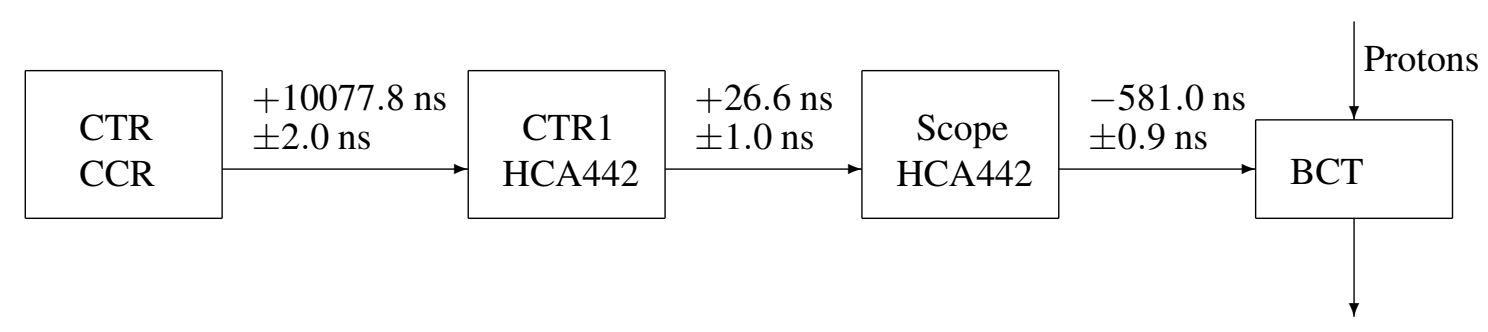

Figure 6. Time delays for the primary beam time stamp.

All stated corrections include propagation delays and signal encoding/decoding latencies. The stated value of $99216.0 \mathrm{~ns}$ is a software delay and has no uncertainty. Figure 6 shows the delay chain. In order to measure the delay between the reference CTR and the CTR1, the CS4000 was transported between them, connecting it to the External Clock Input at one end and the External Start Input at the other. The accuracy of the CS4000 in the time between the two measurements is estimated at better than $2.0 \mathrm{~ns}$ [18]. This delay was also calibrated by means of the two way calibration technique with a reversible fibre path $[6,17,18]$. There is evidence for variations in the cabling delays according to the ambient temperature which can change from day to day [17]. These variations are monitored for the delay between the CTRs by a fibre running in parallel to the cable by comparison with the original calculated value of $10077.8 \mathrm{~ns}$. The estimated yearly variation is less than $2 \mathrm{~ns}$ and is negligible within the time period of data taking of the data discussed in this work. However, the systematic uncertainty is taken conservatively to be $2 \mathrm{~ns}$.

\subsubsection{Secondary beam}

A similar process applies to the timing of the secondary beam (figure 7). It is characterised by four separate delays: (1) the time taken to traverse the hardware between the DDs and the oscilloscope in cavern TZ80, (2) between the oscilloscope trigger and the Control Timing Receiver (CTR2) in cavern TZ80 providing the trigger time stamps (3) the position of the trigger signal within the read-out window, (4) and finally between the CTR2 timebase and the timebase of reference CTR installed in the CCR. The rising edge of the trigger signal triggering the oscilloscope in TZ80, which is time-related to the onset of the SPS kicker magnet, can be placed somewhere around or within the read-out window of the oscilloscope. As the time difference between the start of the read-out window of the BCTF40 and that of the DDs is derived in this section, the relative position of the rising edge of the trigger to the start of the read-out window has to be accounted for. Note, that a different position of the trigger edge would have let to an equivalently differing choice of the software delay explained in section 5.3. The trigger is placed at $2500 \mathrm{~ns}$ after the start of the readout window, hence the window starts 2500 ns earlier. It should be noted that the (4) was measured only with the help of the two way technique, due to the impossibility of transporting the CS4000 to TZ80 cavern.

The delays sum to give the value:

$$
\delta^{\mathrm{DD}}=(-328.2 \pm 0.5+31.1 \pm 1.0-2500.0+29082.9 \pm 2.0) \mathrm{ns}=(26285.8 \pm 2.3) \mathrm{ns} .
$$




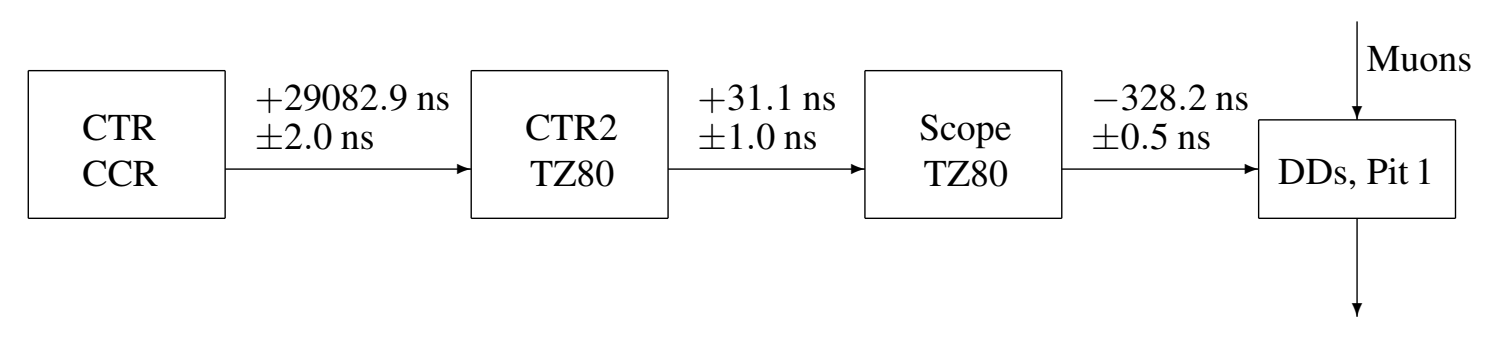

Figure 7. Time delays for the secondary beam time stamp.

\subsection{Calculation of $\delta_{\text {delay }}$}

The time stamps $t_{\mathrm{GPS}}^{\mathrm{BCT}}$ and $t_{\mathrm{GPS}}^{\mathrm{DD}}$ as logged on the TIMBER database [16] for the primary and the secondary beams, respectively, are adjusted by the above offsets to give:

$$
t_{\mathrm{BCT}}=t_{\mathrm{GPS}}^{\mathrm{BCT}}+\delta^{\mathrm{BCT}}
$$

and

$$
t_{\mathrm{DD}}=t_{\mathrm{GPS}}^{\mathrm{DD}}+\delta^{\mathrm{DD}}-30000.0 \mathrm{~ns}
$$

for the start times of the measurement windows. The $-30000.0 \mathrm{~ns}$ is a software delay with no uncertainty and stems from fact that the TIMBER database provides only a certain time window $(12 \mu \mathrm{s})$ out of the total read-out time span. The GPS time stamps logged in the TIMBER database present with a difference of

$$
t_{\mathrm{GPS}}^{\mathrm{BCT}}-t_{\mathrm{GPS}}^{\mathrm{DD}}=(79911.3 \pm 0.5) \mathrm{ns} .
$$

The time difference between the start of the read-out window of the BCTF40 and the start of the read-out window of the DDs is hence:

$$
\begin{aligned}
\delta_{\text {delay }} & =t_{\mathrm{DD}}-t_{\mathrm{BCT}} \\
& =t_{\mathrm{GPS}}^{\mathrm{DD}}+\delta^{\mathrm{DD}}-30000.0 \mathrm{~ns}-\left(t_{\mathrm{GPS}}^{\mathrm{BCT}}+\delta^{\mathrm{BCT}}\right) \\
& =(-79911.3 \pm 0.5+26285.8 \pm 2.3+89692.6 \pm 2.4-30000) \mathrm{ns} \\
& =(6067.1 \pm 3.4) \mathrm{ns} .
\end{aligned}
$$

\section{Data analysis}

The timeline offset $\delta_{\text {offset }}$ of the data within the read-out windows is derived in this section. This is needed, since the windows from BCTF40 and the DDs might not be equally fitted around the signals as depicted in figure 8 . Using $\delta_{\text {offset }}$ and $\delta_{\text {delay }}$ from section 5 , it is possible to measure the ToF from the BCTF40 to the DDs. This serves as a cross-check of the timing of the BCTF40 detector, which was used for the $\mathrm{ToF}_{\nu}$ measurements [6].

The recorded data from the DDs and from the BCTF40 is analysed offline with a custommade analysis making use of the ROOT analysis framework [19]. As described in section 2 the SPS delivers two extractions per cycle. The second extraction has a different shape compared to the first extraction as a result from the SPS filling scheme. Hence, averaging both extractions to 


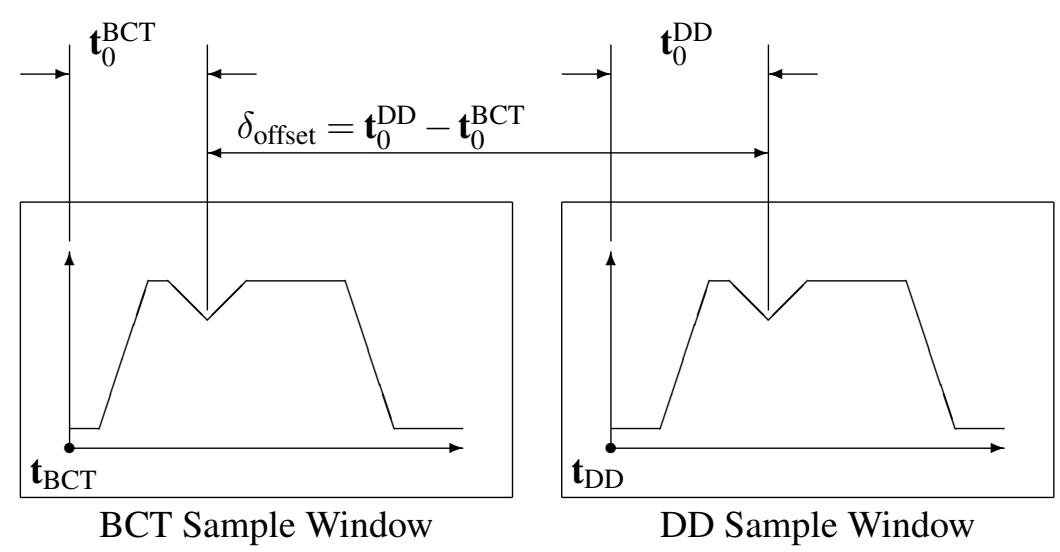

Figure 8. Timeline offset between read-out windows.

form a single pulse is counterproductive, as pulse characteristics like troughs and peaks used later in the analysis would be less pronounced. The analysis presented here focuses on the data obtained from the first extraction to extract a value for $\delta_{\text {offset }}$. After the analysis of the first extraction, the possible improvement of the final results including a full analysis of the second extraction is given.

The sample rate of the presented data is $1 \mathrm{GS} / \mathrm{s}$. Hence, only every fifth data point from the original data taking at $5 \mathrm{GS} / \mathrm{s}$ is used. This reduces the amount of data to be processed by $80 \%$ while keeping the time resolution high enough for the purpose of this analysis. In total 151 spills recorded on 15.11.2011 between 10 a.m. and 11 a.m. (UTC) are taken into account for the presented analysis, of which 119 resulted from non-empty extractions.

Firstly, the detector response from single extractions is shown, then the data treatment is explained, followed by the discussion of the treated data from the three DDs and the BCTF40 individually. The DD and the BCTF40 data is then used to calculate $\delta_{\text {offset }}$.

\subsection{Detector response}

The bare detector response as measured with the oscilloscope within a time window of $12 \mu$ s from a single extraction for the three DDs and the BCTF40 is shown in figure 9. As these plots hold 12000 data points each, the signals appear as a broad band at the given plot resolution. A simple $5 \mathrm{~ns}$ average is added in red to guide the eye. The four detector responses in figure 9 originate from the same extraction. With a sufficiently fast muon detector system, it is reasonable to compare the topology of the signal from the BCTF40 with those from the DDs. A comparison by eye reveals, that certain features of the signal present in all four signals, e.g. the dip flanked by two peaks from $6600 \mathrm{~ns}$ to $8500 \mathrm{~ns}$, as indicated by the arrows. This is the first time that the proton beam structure could be compared to the muon beam structure due to the fact that before the installation of the DDs drift chambers had been used as muon detectors, which have orders of magnitudes larger rise times. Also the shape of the Left detector is similar to those from the Centre detectors, but with reduced intensity and adopted oscilloscope gain. The fact that both technologies, the beam current transformer and the solid state diamond-based ionisation chamber, show similar signals allow the conclusion that the diamond detector system works as expected with an analogue bandwidth of at least as high as the one from the BCTF40 ( 200 MHz). The similarity in shape of the signals then renders a timing analysis possible that utilises the topology of the signals. Note that the BCTF40 

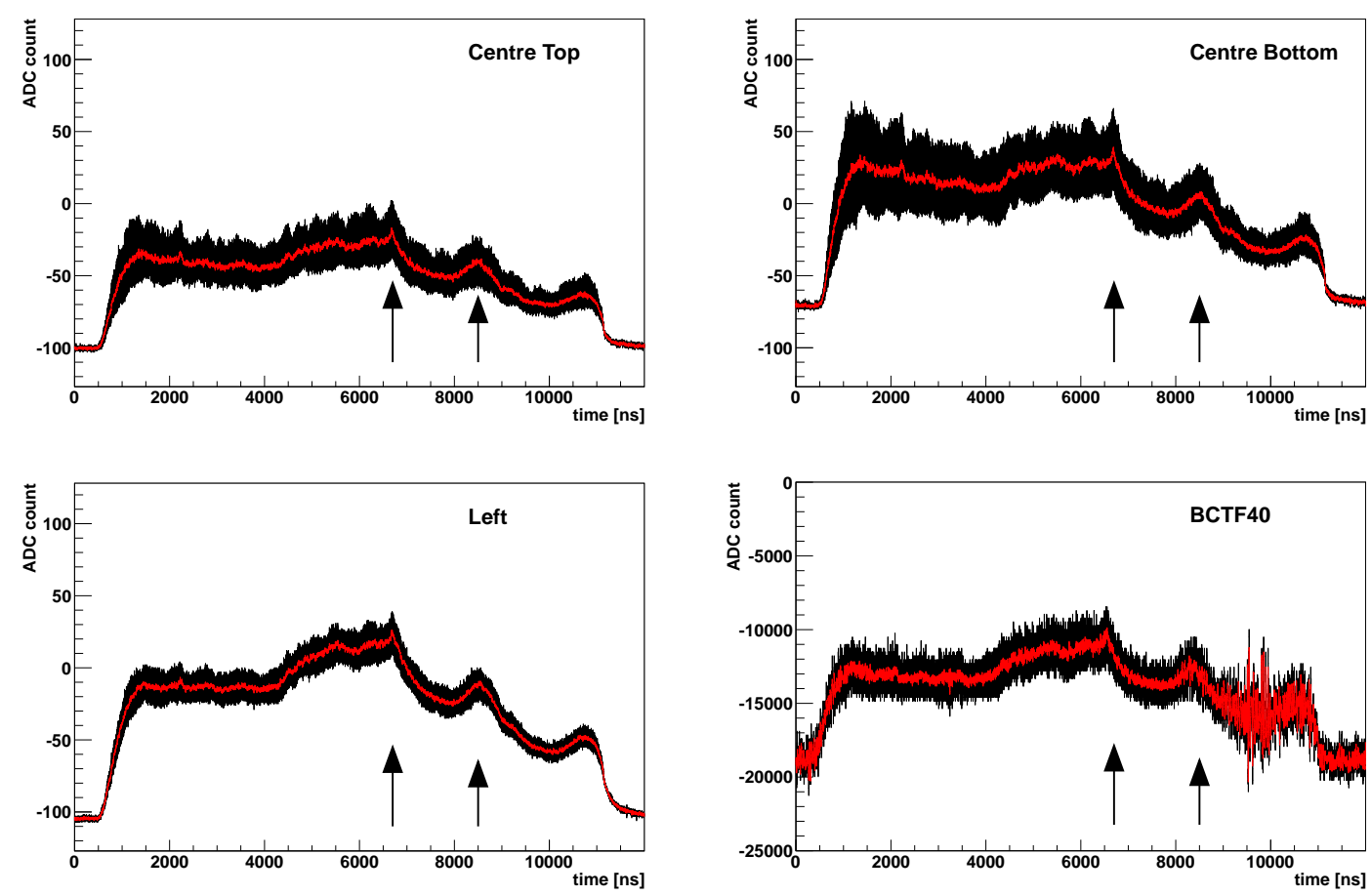

Figure 9. Detector response of a single extraction. In red, a $5 \mathrm{~ns}$ arithmetic average is displayed to guide the eye. The arrows show the same two position in time for all detectors; a very similar structure is observed in all four.

is a beam current transformer and detects the SPS proton beam current, whereas the DDs detect muons originating from pions and kaons produced by SPS protons in the target. However, the DD signals are very similar to the BCTF40 signal.

A zoom from $2800 \mathrm{~ns}$ to $2900 \mathrm{~ns}$ for CT is shown in figure 10. The $5 \mathrm{~ns}(200 \mathrm{MHz}$ ) SPS beam structure is evident. It is worth mentioning that the signal does not return to its baseline at the end of a bunch; each bunch has a tail of the order of few nanoseconds. This leads to a certain pile-up of signal from neighbouring bunches. Therefore, the timing analysis needs to be carried out in such a way that it is not dependent on pile-up.

\subsection{Signal to noise ratio}

The baseline and the baseline noise of the $i$-th extraction, $b_{i}$ and $\sigma_{i}^{\text {noise }}$, measured in ADC counts, are calculated for every non-empty extraction. The mean $\bar{\sigma}^{\text {noise }}$ characterises the mean baseline noise, $\bar{b}$ the mean baseline. Then for each DD as well as for the BCTF40 the average signal amplitude $\langle y\rangle_{i}$ is calculated in the range from $5000 \mathrm{~ns}$ to $6000 \mathrm{~ns}$, where the signal reaches a "flat top". Again, the mean $\overline{\langle y\rangle}$ represents the mean signal amplitude in this region. The two values are used to quantify a signal-to-noise ratio (SNR) for every detector; all numbers are shown in table 3.

The SNR values in table 3 illustrate the difference in signal quality between the DDs and the BCTF40. The DDs deliver an SNR of above 64, in comparison to an SNR of 11 for the BCTF40. The signal quality of the BCTF40 also suffers from oscillations or pick-up noise in the regions from 


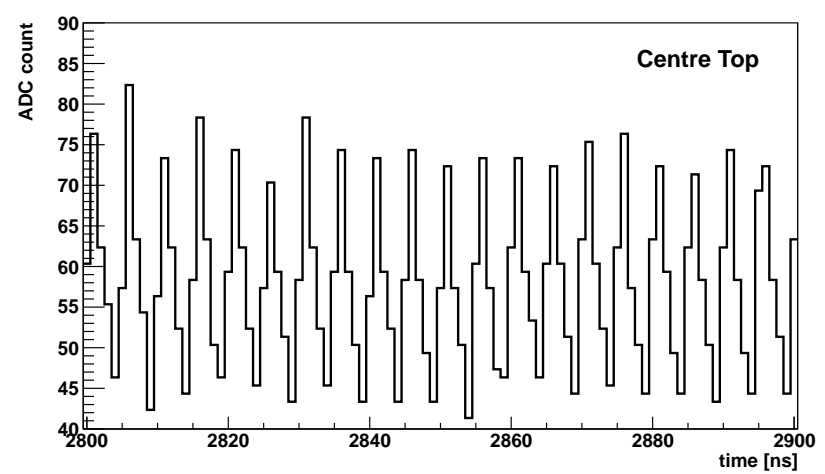

Figure 10. A zoom in the detector response for CT. The baseline is shifted to zero.

Table 3. Characteristic noise and signal values for the DDs and the BCTF40.

\begin{tabular}{|l|c|c|c|c|}
\hline & $\mathrm{BCT}$ & $\mathrm{CT}$ & $\mathrm{CB}$ & $\mathrm{L}$ \\
\hline $\bar{\sigma}^{\text {noise }} / \mathrm{ADC}$ counts & 650 & 1.09 & 1.17 & 1.43 \\
$\overline{\langle y\rangle} / \mathrm{ADC}$ counts & -11570 & -30.35 & 23.72 & 8.16 \\
$\bar{b} / \mathrm{ADC}$ counts & -18791 & -100.45 & -71.0 & -104.90 \\
\hline SNR & 11.1 & 64.3 & 81.0 & 79.1 \\
\hline
\end{tabular}

$0 \mathrm{~ns}$ to $2000 \mathrm{~ns}$, and from $8000 \mathrm{~ns}$ to $12000 \mathrm{~ns}$. A frequency analysis of these parts revealed a major contribution at $25 \mathrm{MHz}$. This pick-up is common to all recorded extractions for the BCTF40. These parts of the signal are therefore disregarded in the timing analysis. CB and L have better SNRs than the CT for two reasons: (1) the electrode areas of $\mathrm{CB}$ and $\mathrm{L}$ are larger than that of CT leading to a larger signal and (2) the fact that the noise is not dominated by the detectors capacity but rather by the least-significant-bit noise of the oscilloscope.

\subsection{Time resolution and phase shift}

The single-pulse time resolution of DDs in combination with fast electronics has often been proven to be better than $1 \mathrm{~ns}$ for MIPs, cf. e.g. [20]. This holds true as well for the DDs used in this analysis and has been tested in the laboratory before installation. For a higher signal charge than the charge produced by a MIP, a better time resolution is expected. From figure 10, partial rise times for every bunch of $t_{\text {rise }} \leq 2 \mathrm{~ns}$ over $30 \mathrm{ADCs}$ can be concluded, and hence a slope $s$ of $s>15 \mathrm{ADC} / \mathrm{ns}$. Therefore, the time resolution $\sigma_{\mathrm{t}}=\frac{\bar{\sigma}^{\text {noise }}}{s}<0.1 \mathrm{~ns}$.

The difficulty in this analysis is the complexity of the signal; the uncertainty of the point in time of the arrival of the first bunch at the DDs is not dominated by the time resolution of the DDs themselves, but by the long rise time $(1 \mu \mathrm{s})$ of the kicker magnet resulting in a steadily increasing muon intensity per bunch arriving at the DDs during the rising edge. However, the jitter of the trigger signal is small, hence it is sufficient to find the modulo $5 \mathrm{~ns}$ shift for each extraction. This shift can then be applied to each extraction. Thereby, the phases of the extractions are aligned 


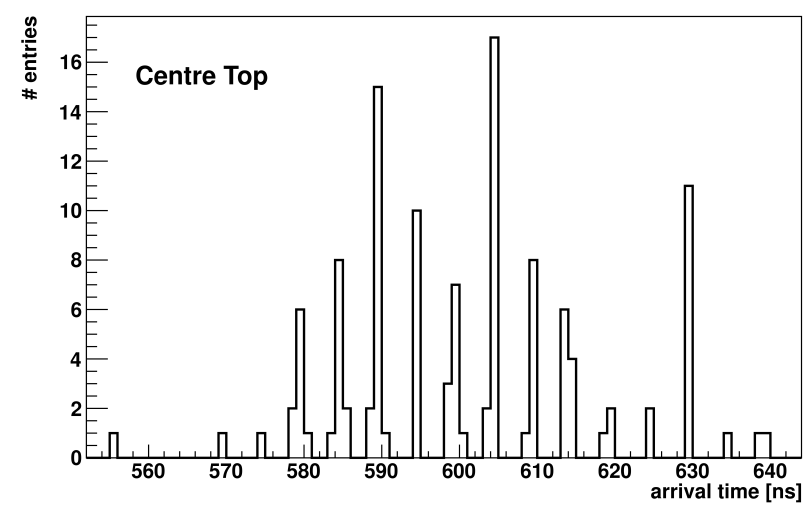

Figure 11. The corrected arrival time for the 119 events non-empty extractions.

such that an equivalent point in time is found. The modulo $5 \mathrm{~ns}$ shift is found by analysing the signal within the global rising edge - as opposed to a local one for a single bunch — from $800 \mathrm{~ns}$ to $1000 \mathrm{~ns}$. The SPS beam structure can easily be found by comparing five different sums $\Sigma^{(n)}$, where each sum represents a sum over signal amplitude values $y_{\mathrm{DD}}(t)$ separated by $5 \mathrm{~ns}$ with an offset of $n=0,1,2,3,4$ ns: $\sum^{(n)}=\sum_{i} y_{\mathrm{DD}}(5 i+n)$. The largest sum finds the phase $\varphi_{\mathrm{i}}$ of the maximum within the $5 \mathrm{~ns}$ SPS beam structure. The resulting histogram of the $\varphi_{\mathrm{i}}$-corrected arrival time is shown in figure 11. The evident $5 \mathrm{~ns}$ structure of the time of arrival shows the aptitude of this approach. The spread in the corrected arrival time is, again, cased by the long rising edge.

\subsection{Data treatment}

\subsubsection{Averaging}

In order to make a statement about the CNGS timing at CERN, the 119 non-empty signals are combined to form an average signal. This averaging makes the analysis robust against fluctuations in the single extractions. In order to average the signals correctly, the phase $\varphi_{i}$ is used. The timeline of every single extraction is shifted by $-\varphi_{i}$ in order to ensure an aligned phase. For simplicity the baseline was shifted to zero. The average pulse values $y_{\text {avg }}(t)=\sum_{i} y_{i}(t) / 119-b$, with the baseline $b$ from table 3 . The average signal for CT is shown in figure 12 .

The difference in average shift $\Delta \bar{\varphi}=\bar{\varphi}_{\mathrm{DD}}-\bar{\varphi}_{\mathrm{BCT}}$ is determined for each DD with respect to the BCTF40. These average shift differences are much smaller than the errors resulting from other uncertainties, and can hence be omitted.

Note that the signal amplitude of the average pulse varies about 30 ADCs on top of the DC part, about as much as for the single pulse, compare figure 10 and figure 12 (right). This is to be expected if the phase-finding algorithm is working, and hence the phases are not smeared out, and if the extractions are sufficiently alike.

\subsubsection{Filtering and normalisation}

The averaged signal is now filtered in order to get rid of the SPS beam structure which facilitates the later timing analysis. The applied filter method is a raised-cosine filter of width $w$, which belongs 

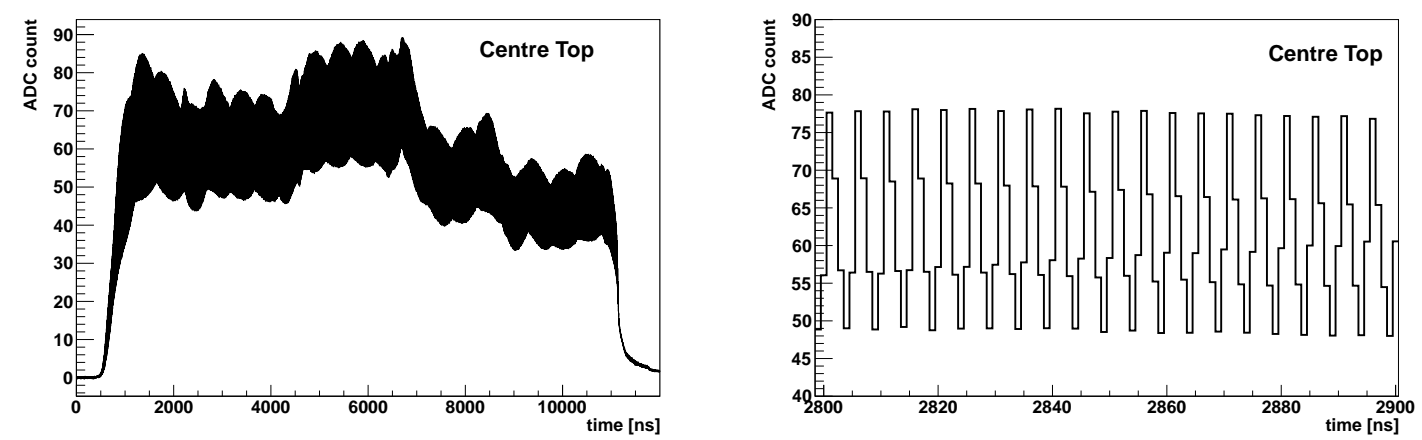

Figure 12. Overview (left) and zoom (right) of the averaged signal for CT.

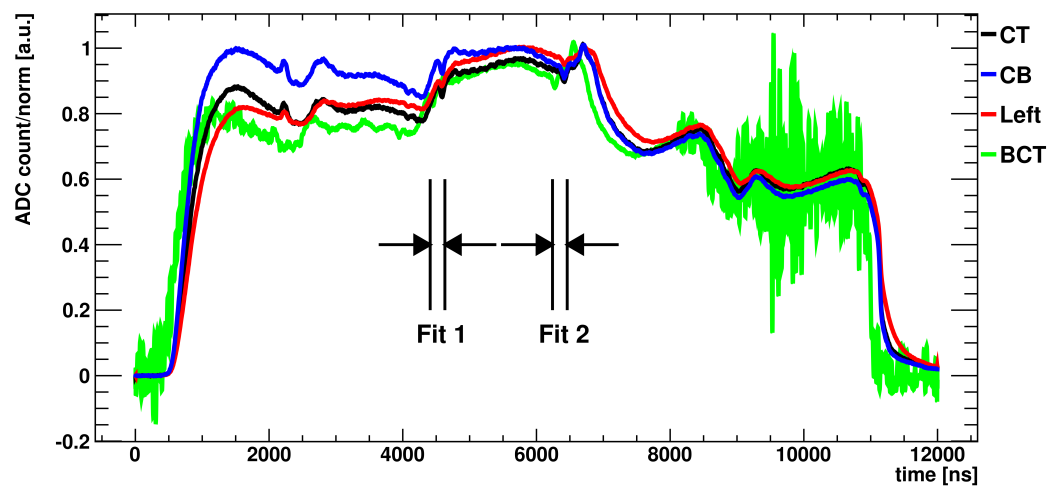

Figure 13. The averaged, filtered, and normalised detector responses are displayed for the three DDs and the BCTF40.

to the class of Finite Impulse Response filters. The applied equation for filtering is:

$$
y_{\text {avg,filterd }}(t ; w)=\sum_{j=-w / 2}^{w / 2} y_{\text {avg }}(t+j) \times(1+\cos (2 \pi(j / w))),
$$

and the averaged, filtered signals with $w=15 \mathrm{~ns}$ for CT, CB, L, and BCTF40 are shown in figure 13. Each DD signal is normalised to its average amplitude between $6650 \mathrm{~ns}$ and $6750 \mathrm{~ns}$, the section of highest signal amplitude featuring a sharp peak. The same is done for the BCTF40, but with the average amplitude taken between $6510 \mathrm{~ns}$ and $6610 \mathrm{~ns}$. The windows for the calculation of the average amplitude do not overlap for the DDs and the BCTF40. A difference of $140 \mathrm{~ns}$ was chosen due to obvious indications of an offset between the starting point of the read-out windows, that is e.g. the sharp peak appearing $140 \mathrm{~ns}$ earlier in the BCTF40 data than in the DDs data as well as the position of the rising/falling edge at the the start/end of the extractions.

\subsection{Calculation of $\delta_{\text {offset }}$}

Figure 8 explains the timeline offset $\delta_{\text {offset }}$ between the signals within their read-out windows. The averaged and filtered pulses are used to determine $\delta_{\text {offset }}$ in two different ways. The "fitting method" 


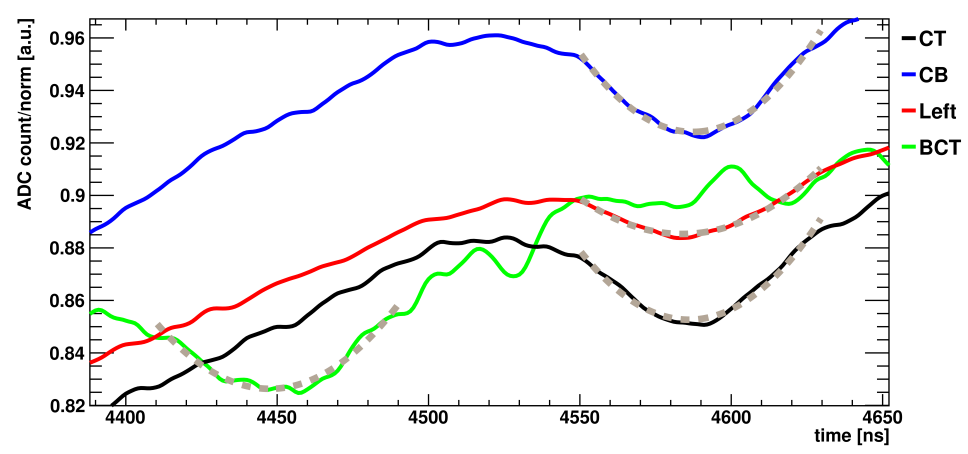

Figure 14. The averaged, filtered detector response (solid lines) around a trough and the corresponding parabolic fits (dashed) are shown for one fit region.

uses the topology of the signal at two different positions applying a fit to the detector responses over a sample section of $80 \mathrm{~ns}$ width. The chosen sections feature a trough and are indicated in figure 13 with "Fit 1" and "Fit 2". This method is referred to as local. A method that uses a much wider section, is the cross-correlation method. This calculates the cross-correlations between two signals over a number of microseconds. Therefore, this method is referred to as global.

\subsubsection{The local method}

The fitting method exploits a rather simple approach of fitting a second order polynomial to an apparent trough in the detector responses,

$$
y_{\text {fit }}(t)=a\left(t-t_{0}\right)^{2}+c
$$

within a section of $80 \mathrm{~ns}$ width. The $t_{0}$ defines the lowest point of the trough and is readily determined for all four detector signals. The method is applied in two regions: from $4550 \mathrm{~ns}$ to $4630 \mathrm{~ns}$ (Fit 1), shown in figure 14, and from $6380 \mathrm{~ns}$ to $6460 \mathrm{~ns}$ (Fit 2) for the DDs, but $140 \mathrm{~ns}$ earlier for both fits for the BCTF40. As well by eye it is visible, that a steep trough is apparent in all four detector signals alike, but about $140 \mathrm{~ns}$ earlier in time for the BCTF40. Each fit then yields a $t_{0}$. The values are collected in table 4 (top). The difference between the $t_{0}$ for the DDs and the BCTF40 form $\delta_{\text {offset }}, \delta_{\text {offset }}=t_{0}^{\mathrm{DD}}-t_{0}^{\mathrm{BCT}}$, see table 4 (bottom). Note that pile-up adds to $c$, but does not affect $t_{0}$. Hence, this approach is robust against pile-up.

As the result of this method, the weighted mean and error of the six $\delta_{\text {offset }}$ values are quoted:

$$
\delta_{\text {offset }}^{\mathrm{fit}}=(140.0 \pm 2.5) \mathrm{ns}
$$

\subsubsection{The global method}

This method employs a one-parameter likelihood function (LF) between two signals to form a likelihood estimator $\hat{\Theta}$, e.g. between CT and BCTF40: $\hat{\Theta}(\tau ; \mathrm{CT}, \mathrm{BCT})=\mathrm{LF}\left(f_{\mathrm{CT}}(t), f_{\mathrm{BCT}}(t+\tau)\right)$, in order to find a possible offset between them. If a signal coming from either a DD or from the BCTF40 is correlated with itself, it is called an auto-estimator $\left(\hat{\Theta}_{a}\right)$. A cross-estimator $\left(\hat{\Theta}_{x}\right)$ 
Table 4. Fit results for $t_{0}$ and the resulting $\delta_{\text {offset }}$.

\begin{tabular}{|c|c|c|c|c|c|}
\hline & & BCT & CT & CB & L \\
\hline trough 1 & $t_{0} / \mathrm{ns}$ & $4446.9 \pm 0.5$ & $4586.1 \pm 0.5$ & $4586.4 \pm 0.5$ & $4582.6 \pm 1.1$ \\
trough 2 & $t_{0} / \mathrm{ns}$ & $6272.8 \pm 0.8$ & $6413.1 \pm 0.8$ & $6415.6 \pm 0.8$ & $6420.5 \pm 1.4$ \\
\hline & & & CT-BCT & CB-BCT & L-BCT \\
\hline trough 1 & $\delta_{\text {offset }} / \mathrm{ns}$ & & $139.2 \pm 0.7$ & $139.5 \pm 0.7$ & $135.7 \pm 1.2$ \\
trough 2 & $\delta_{\text {offset }} / \mathrm{ns}$ & & $140.3 \pm 1.1$ & $142.8 \pm 1.1$ & $147.7 \pm 1.6$ \\
\hline
\end{tabular}

is the result of a correlation of two signals stemming from different detectors. In order to find the $\delta_{\text {offset }}$, the correlation is calculated as a function of a parameter $\tau$ which describes a time shift between the time arguments of the correlated functions, hence $\hat{\Theta}_{x}\left(\tau ; f_{1}, f_{2}\right)=\operatorname{LF}\left(f_{1}(t), f_{2}(t+\tau)\right)$, and $\hat{\Theta}_{a}\left(\tau ; f_{1}\right)=\operatorname{LF}\left(f_{1}(t), f_{1}(t+\tau)\right)$. The auto-estimator is used to check that the used method yields reasonable results in terms of topology and amplitude. The expected result for the $\hat{\Theta}_{a} \mathrm{~s}$ is a minimum at $\tau=0$. Furthermore, the depth of the minimum for the $\hat{\Theta}_{a}$ can be compared to that of the $\hat{\Theta}_{x}$; the latter is expected to be shallower than the former. The absolute value at the minimum of $\hat{\Theta}_{a}(\tau)$ should be zero for all auto-estimators.

The chosen likelihood function uses the absolute difference in first derivative at a given point in time for the two signals under test, hence $\left|f_{1}^{\prime}(t)-f_{2}^{\prime}(t+\tau)\right|$. This difference is calculated for every point in time $t$ in a specified range, i.e. from $t_{\text {start }}$ to $t_{\text {end }}$. The absolute values are being summed; the sum forming the cross-estimator value $\hat{\Theta}_{x}(\tau)$ in case different functions are correlated, or the auto-estimator value $\hat{\Theta}_{a}(\tau)$ in case a function was correlated with itself. This procedure is then repeated for every $\tau$ ranging from $-500 \mathrm{~ns}$ to $+500 \mathrm{~ns}$ in $1 \mathrm{~ns}$-steps. The position of the minimum of $\hat{\Theta}_{x}(\tau ; \mathrm{DD}, \mathrm{BCT})$ is the offset between the recording windows. One minimum is found for every DD-BCTF40-pair. The full formula is:

$$
\hat{\Theta}_{x}\left(\tau ; f_{1}, f_{2}\right)=\sum_{t=t_{\text {start }}}^{t_{\text {end }}}\left|f_{1}^{\prime}(t)-f_{2}^{\prime}(t+\tau)\right|, \tau=-500 \mathrm{~ns}, \ldots, 500 \mathrm{~ns}
$$

and equivalently for $\hat{\Theta}_{a}(\tau)$. The local derivative for a progression - the recorded signal is a progression with finite time steps rather than a continuous function - is the difference of two neighbouring points: $f^{\prime}=\frac{f(t)-f(t+1 \mathrm{~ns})}{\Delta t}$. Here $\Delta t=1 \mathrm{~ns}$. It is stressed that the "standard" crosscorrelation function $(f * g)(\tau)=\sum f_{1}(t) f_{2}(t+\tau)$ has not been chosen as the LF. The crosscorrelation is not independent of the absolute values of the tested correlated signals, hence global maxima might appear due to changes in amplitude values, and not due to a better match. Therefore, in the presented analysis the difference rather than the product of two functions is chosen for the LF in order to avoid this dependence. Then, the first derivative is chosen as the function ought to be correlated. This has the advantage that the minimum of the likelihood estimator is more pronounced, as can easily be shown. Additionally, differentiation attenuates the low frequency spectrum where most of the spectral differences between detectors are located, e.g. possible skin effect on the cables, different detector capacitances, pile-up and possible offset on the signals. Both auto- and cross-estimators have been calculated in the range from $t_{\text {start }}=4000 \mathrm{~ns}$ to $t_{\text {end }}=8000 \mathrm{~ns}$. 

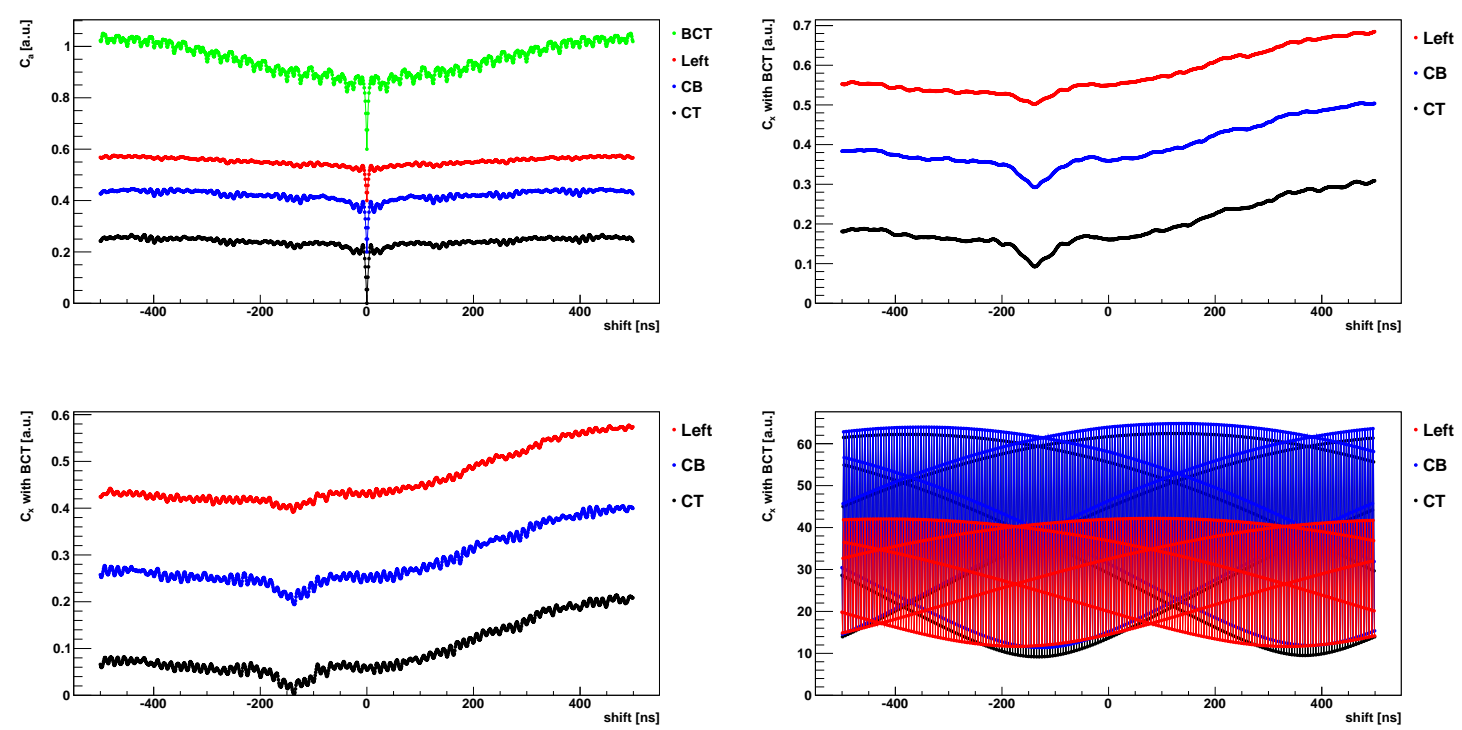

Figure 15. The likelihood auto-estimators $\hat{\Theta}_{a}(\tau)$ are shown for $w=15$ for the four detectors separately, with all their minima at $0 \mathrm{~ns}$ (top left). The likelihood cross-estimators $\hat{\Theta}_{x}(\tau ; \mathrm{DD}, \mathrm{BCT})$ between each DD and the BCTF40 for $w=30 \mathrm{~ns}$ and $w=15 \mathrm{~ns}$ (top right, bottom left) have their minima around $-138 \mathrm{~ns}$. The likelihood cross-estimators $\hat{\Theta}_{x}(\tau ; \mathrm{DD}, \mathrm{BCT})$ for $w=5 \mathrm{~ns}$ (bottom right) shows no pronounced minimum.

The calculated auto-estimators are shown in figure 15 (top left) for a filter width of $w=15 \mathrm{~ns}$. The minima of the $\hat{\Theta}_{a} \mathrm{~s}$ are more pronounced then the minima of the $\hat{\Theta}_{x} \mathrm{~s}$, as expected, and their position are at $0 \mathrm{~ns}$ as expected from an auto-correlation. Also, their position are at $\tau=0$ independent of $w$ (not shown). Furthermore, the absolute value at $\tau=0$ is exactly zero, but the functions in figure 15 (top left) are drawn with an offset for better visibility.

The cross-estimators of the BCTF40 signal with each DD signal separately are shown in figure 15 (top right, bottom) as a function of $\tau$ for three different filter widths. In order to avoid an overlay of the different cross-correlation graphs, arbitrary offsets have been added to the $\hat{\Theta}_{x} \mathrm{~s}$. The position of the minima for $\hat{\Theta}_{x}(\mathrm{CT}, \mathrm{BCT}), \hat{\Theta}_{x}(\mathrm{CB}, \mathrm{BCT})$, and $\hat{\Theta}_{x}(\mathrm{~L}, \mathrm{BCT})$, i.e. the calculated $\delta_{\text {offset }} \mathrm{s}$, are listed in table 5. Their mean and error are $(138.1 \pm 1.2) \mathrm{ns}$. It is evident from the lower right picture of figure 15, that a raised cosine filter of $5 \mathrm{~ns}$ width is not sufficient to filter out the SPS bunch structure from the detector response. This results in a larger variation in the cross-estimator $\hat{\Theta}_{x}$ value due to the matching or non-matching bunches depending an the $\tau$ under test (45 a.u. amplitude variation within $5 \mathrm{~ns}$ - the high frequency part) than a match from the overall extraction structure (around 10 a.u. over $200 \mathrm{~ns}$ - the "envelope part"). Additionally, the $\hat{\Theta}_{x}$ s between different DDs have been checked. The positions of the minima are also located at $\tau=0$, which further proves the reliability of this method.

As the result of the likelihood estimator method, the mean and standard deviation of the nine $\delta_{\text {offset }}$ values are quoted:

$$
\delta_{\text {offset }}^{\hat{\Theta}_{x}}=(138.1 \pm 1.2) \mathrm{ns} .
$$


Table 5. The position of the minimum of likelihood cross-estimator $\hat{\Theta}_{x}(\mathrm{DD}, \mathrm{BCT})$ between the BCTF40 with the individual DDs.

\begin{tabular}{|l|c|c|c|}
\hline & \multicolumn{3}{|c|}{$\min \left(\hat{\Theta}_{x}(\mathrm{DD}, \mathrm{BCT})\right)$} \\
& $\mathrm{DD}=\mathrm{CT}$ & $\mathrm{DD}=\mathrm{CB}$ & $\mathrm{DD}=\mathrm{L}$ \\
\hline$\delta_{\text {offset }} / \mathrm{ns}$ for $w=15 \mathrm{~ns}$ & 137 & 136 & 139 \\
$\delta_{\text {offset }} / \mathrm{ns}$ for $w=20 \mathrm{~ns}$ & 138 & 137 & 138 \\
$\delta_{\text {offset }} / \mathrm{ns}$ for $w=30 \mathrm{~ns}$ & 139 & 139 & 140 \\
\hline
\end{tabular}

\section{Results}

In order to verify the CNGS timing at CERN, the ToF for charged particles from the beam current transformer BCTF40 to the diamond detectors downstream of the CNGS target was measured and compared to its nominal value. The measured ToF uses the total delay between the start of the read-out windows $\delta_{\text {delay }}$ and the offset $\delta_{\text {offset }}$ of the timelines within the recording windows, hence $\mathrm{ToF}=\delta_{\text {delay }}+\delta_{\text {offset. }}$. As presented in section 5, the time difference between the start of the recording window of the BCTF40 and the recording window of the DDs is

$$
\delta_{\text {delay }}=(6067.1 \pm 3.4) \mathrm{ns} .
$$

In section $6, \delta_{\text {offset }}$ was calculated based on two different methods to be

$$
\delta_{\text {offset }}^{\text {fit }}=(140.0 \pm 2.5) \mathrm{ns} \quad \text { and } \quad \delta_{\text {offset }}^{\hat{\Theta}_{x}}=(138.1 \pm 1.2) \mathrm{ns}
$$

using the fitting method and the likelihood cross-estimator method, respectively. Although derived on very different parts of the signal, each method's result is consistent with the other within their precision. No additional source of a significant systematic uncertainty is known. Neither do the results suggest one. The measured $\mathrm{ToF}$ is hence

$$
\mathrm{ToF}_{\text {fit }}=(6207.1 \pm 4.2) \mathrm{ns} \text { and } \mathrm{ToF}_{\hat{\Theta}_{x}}=(6205.2 \pm 3.6) \mathrm{ns}
$$

for the fitting method and for the likelihood cross-estimator method, respectively.

A cross-check of the data stemming from the second extraction was done, and no significant difference to the $\delta_{\text {offset }}$ of the first extraction is found. A full analysis of the second extraction would improve the uncertainties of $\delta_{\text {offset }}^{\hat{\Theta}_{x}}$ and $\delta_{\text {offset }}^{\text {fit }}, \sigma_{\delta_{\text {offset }}^{\hat{\Theta}_{x}}}$ and $\sigma_{\delta_{\text {offset }}^{\text {fit }}}$ respectively, roughly by a factor of $\sqrt{2}$. Since the uncertainty of $\delta_{\text {delay }}$ is larger than $\sigma_{\delta_{\text {offiset }}^{\hat{\theta}_{x}}}$ and $\sigma_{\delta_{\text {offset }}^{\text {fit }}}$ and since the ToF is the sum of

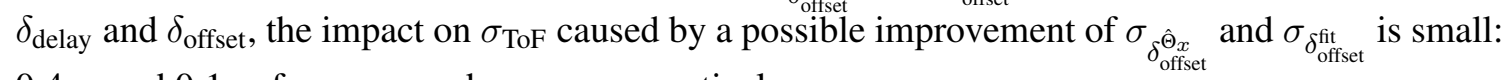
$0.4 \mathrm{~ns}$ and $0.1 \mathrm{~ns}$ for $\sigma_{\mathrm{ToF}_{\text {fit }}}$ and $\sigma_{\mathrm{ToF}_{\hat{\Theta}_{x}}}$, respectively.

The value for the nominal ToF combines the travelled distance of $(1859.95 \pm 0.02) \mathrm{m}$, as measured by the CERN GEO survey, and the combined velocity $v$ of the original proton and its production/decay products. The SPS protons have a momentum of $400 \mathrm{GeV} / c$, the pions and kaons an average momentum of $35 \mathrm{GeV} / c$, and hence the muons have an average momentum of $17 \mathrm{GeV} / c$. 
Under the assumption of not-completely relativistic muons, a ToF of

$$
\mathrm{ToF}_{\mathrm{nom}}=\frac{1859.95 \mathrm{~m}}{v}=(6205.3 \pm 1.7) \mathrm{ns},
$$

is found, where the uncertainty is dominated by the decay position of the pions/kaons within the decay tube. The $1.7 \mathrm{~ns}$ reflect the spread in decay position of $68 \%$ of the pions/kaons decaying in the decay tube, centred midway within the tube, and the resulting difference in arrival time. $\mathrm{ToF}_{\text {nom }}$ lies well within the errors of $\mathrm{ToF}_{\text {fit }}$ and $\mathrm{ToF}_{\hat{\Theta}_{x}}$. Under the assumption of completely relativistic muons, $\mathrm{ToF}_{\mathrm{nom}}=\frac{1859.95 \mathrm{~m}}{c}=(6204.1 \pm 1.7) \mathrm{ns}$.

\section{Conclusion}

As a result of the neutrino time-of-flight publication of OPERA in 2011, polycrystalline CVD diamond detectors were installed in the secondary beam line downstream from the CNGS target in order to measure the time structure of the muon spill. This allows for an independent verification of the CNGS timing at CERN, which was previously only based on the measurements of the protons passing through a fast beam current transformer (BCTF40) before they hit the CNGS target. The distance between the two detector positions was carefully measured as well as all the time delays from the detection of the particles until the signal was registered. Detailed analysis of the signal shape was performed. The nominal time-of-flight of the protons and their secondary particles produced in the CNGS target travelling at almost the speed of light between the BCTF40 and the diamond detectors was compared with the measured time-of-flight of the charged particles passing through these detectors. The nominal time-of-flight lies well within the uncertainty of the measured time-of-flight. The results show that the CNGS time measurements performed at CERN using the BCTF40 and the diamond detectors are consistent.

\section{Acknowledgments}

The authors would like to thank the CERN service groups for the fast and efficient realisation of the diamond detector installation.

\section{References}

[1] K. Elsener et al., The CERN neutrino beam to Gran Sasso (NGS) : conceptual technical design, CERN 98-02, INFN/AE-98/05 (1998).

[2] Borexino collaboration, G. Alimonti et al., The Borexino detector at the Laboratori Nazionali del Gran Sasso, Nucl. Instrum. Meth. A 600 (2009) 568, http://borex.lngs.infn.it/.

[3] ICARUS collaboration, ICARUS: a proposal for the Gran Sasso Laboratory, INFN/AE-85/7, Frascati Italy (1985), http://icarus.lngs.infn.it/.

[4] LVD collaboration, C. Alberini et al., The large volume detector (LVD) of the Gran Sasso laboratory, Nuovo Cim. C 9 (1986) 237, http://www.nu.to.infn.it/exp/all/lvd/.

[5] OPERA collaboration, M. Guler et al., An appearance experiment to search for $\nu_{\mu} \nu_{\tau}$ oscillations in the CNGS beam: experimental proposal, CERN/SPSC 2000-028 (2000), http://operaweb.lngs.infn.it/. 
[6] OPERA collaboration, T. Adam et al., Measurement of the neutrino velocity with the OPERA detector in the CNGS beam, arXiv:1109.4897.

[7] H. Jacob et al., A $40 \mathrm{MHz}$ Bunch by Bunch Intensity Measurement for the CERN SPS and LHC, CERN-AB 2003-056 BDI (2003).

[8] A. Ferrari et al., FLUKA: A multi-particle transport code (program version 2005), CERN-2005-010, SLAC-R-773, INFN-TC-05-11 (2005);

G. Battistoni et al., The FLUKA code: description and benchmarking, AIP Conf. Proc. 896 (2007) 31.

[9] A. Ferrari et al., An updated Monte Carlo calculation of the CNGS neutrino beam, CERN-AB-Note-2006-038 (2007).

[10] D. Autiero et al., The CNGS neutrino beam: status, Nucl. Phys. Proc. Suppl. 188 (2009) 188.

[11] Element Six, http://www.e6.com/.

[12] Diamond Detectors Ltd., http://www.diamonddetectors.com/.

[13] Cividec Instrumentation, http://www.cividec.at/.

[14] E. Griesmayer et al., Test of a Diamond Detector Using Unbunched Beam Halo Particles, CERN-ATS-2010-027 (2010).

[15] http://www.drakact.com/.

[16] http://hc-logging.web.cern.ch/lhc-logging/.

[17] P. Alvarez, J. Serrano, http://www.ohwr.org/documents/112.

[18] P. Alvarez, http://www.ohwr.org/documents/116.

[19] R. Brun and F. Rademakers, ROOT An object oriented data analysis framework, Nucl. Instrum. Meth. A 389 (1997) 81.

[20] D. Dobos and H. Pernegger, Diamond pixel modules and the ATLAS beam conditions monitor, Nucl. Instrum. Meth. A 628 (2011) 246. 